\section{P2-296 DIFFERENCES IN SOCIOECONOMIC STATUS, SPREAD OF DISEASE, RURAL RESIDENCE AND ACCESS TO SURGERY DO NOT EXPLAIN LOWER SURVIVAL FROM BREAST CANCER FOR ABORIGINAL WOMEN}

doi:10.1136/jech.2011.142976k.29

R Supramaniam,* D O'Connell. Cancer Council NSW, Sydney, New South Wales, Australia

Introduction We wanted to investigate any differences in breast cancer survival for Aboriginal women compared with nonAboriginal women in New South Wales, Australia (NSW).

Methods All women diagnosed with primary invasive breast cancer in NSW in 1994-2002 $(n=31525)$ were probabilistically linked with all NSW hospital admissions. 296 women identified as Aboriginal at any hospital admission. Proportional hazards models were used to investigate the time to first surgery and to analyse cause-specific survival adjusting for differences in comorbidities, diagnosis age, disease spread, diagnosis year, rural residence and socioeconomic status.

Results Aboriginal women in NSW had similar times to their first surgery as non-Aboriginal women (HR $0.74,95 \%$ CI 0.51 to 1.06 ) after adjusting for age, year of diagnosis, disease spread, socioeconomic status, rural residence and comorbidities. However, Aboriginal women were $53 \%$ more likely to die of their breast cancer (HR 1.53, 95\% CI 1.19 to 1.97), after adjusting for whether they had surgery, diagnosis age, disease spread, diagnosis year, socioeconomic status, rural residence and comorbidities.

Conclusions Differences in breast cancer survival in NSW for Aboriginal and non-Aboriginal women were not totally explained by demographic, disease or access to surgical treatment differences. Residual differences in breast cancer survival maybe due to differences in uptake of adjuvant therapies including chemotherapy and radiotherapy, unmeasured comorbidities or cultural barriers to accessing optimal cancer care. Overall breast cancer treatment and outcomes were better than for other cancers and we will investigating the reasons for this.

\section{P2-297 SURVIVAL ANALYSIS OF DIALYSIS PATIENTS IN THE BRAZILIAN HEALTH SYSTEM}

doi:10.1136/jech.2011.142976k.30

D Szuster, ${ }^{*}$ W Caiaffa, E I Andadre, F Acurcio, M Cherchiglia. Universidade Federal de Minas Gerais, Belo Horizonte, Minas Gerais, Brazil

Introduction This study performed survival analysis on patients that had commenced "Haemodialysis (HD) and Peritoneal Dialysis (DP) renal replacement therapy (RRT)" on the National Public System in Brazil.

Method The following criteria were used to select patient records: patients admitted between 2002 and 2003 with at least three consecutive months of treatment and $>18$ years of age. Independent variables tested were gender, age, region of residence, basic cause of kidney failure, Human Development Index (IDH) of the year 2000. A proportional hazards model to investigate the factors associated with death was used.

Results There were 31298 patients. The majority were on HD, average age of 54 years, and residing in the Southeast region. The average IDH for the residence cities of the patients was 0.78 . The main IRCT diagnosis was hypertension. The final model showed that the following variables were associated with a greater probability of death after 3 years of follow-up: female gender; age over 55 years; primary diagnosis of mellitus diabetes; initiated on DP; not residing in the southeast region. Residing in cities with greater IDH was related to a reduced risk of death. The adjusted estimated risk, for the 3-year treatment, was $\mathrm{HR}=1.17$, in favour of $\mathrm{HD}$. The evaluation of risk between the modalities has shown that age is an important factor for a greater risk on DP, independently of gender and IRCT cause.

Conclusion The findings were corroborated by literature data that indicate that older patients have a worse prognosis when undergoing DP.

\section{P2-298 MEAT DERIVED MUTAGENIC ACTIVITY AND THE RISK OF COLORECTAL CANCER}

doi:10.1136/jech.2011.142976k.31

${ }^{1,2} \mathrm{~S} \mathrm{M}$ Tabatabaei, ${ }^{*} 1 \mathrm{~J}$ Heyworth, ${ }^{1} \mathrm{~L}$ Fritschi, ${ }^{1} \mathrm{M}$ Knuiman. ${ }^{1}$ University of Western Australia, Western Australia, Australia; ${ }^{2} Z a h e d a n$ University of Medical Sciences, Zahedan, Iran

Introduction Colorectal cancer (CRC) is an important cause of morbidity and mortality. High intake of meat has been associated with increased risk of CRC in some studies but results are inconsistent. Instead of examining meat intake it may be appropriate to examine total meat-derived mutagenic activity (MDMA), which incorporates the mutagenic activity from identified and other yet unidentified chemical compounds in cooked meat.

Methods This case-control study used data from the Western Australian Bowel Health Study. It included 567 cases and 713 age and sex frequency matched controls, aged between 41 and 80 years. Meat consumption information was collected via selfadministered questionnaires. Exposure to MDMA and predicted heterocyclic amine -derived mutagenic activity (PHDMA) was estimated by linking the meat data into a carcinogen database (CHARRED). The data were analysed using multivariable logistic regression.

Results ORs for increasing quartiles of MDMA indicated no association with CRC. Although the ORs for comparing subjects in the higher quartiles of PHDMA based on total and red meat consumption with lower quartiles of intake were less than one, none of these relationships were statistically significant. However, the PHDMA from white meat was associated with a non-significant increase in the risk of CRC (OR high compared with lowest quartile $=1.17,95 \%$ CI 0.83 to $1.64, \mathrm{p}>0.30$ ).

Conclusion Our study did not support the association between exposure to MDMA and the risk of CRC.

\section{P2-299 MEN'S PERCEPTIONS AND EXPERIENCES OF THE EARLY DETECTION OF PROSTATE CANCER: A QUALITATIVE STUDY USING GROUNDED THEORY APPROACH}

doi:10.1136/jech.2011.142976k.32

${ }^{1} \mathrm{~A}$ Taghipour, ${ }^{2}{ }^{2} \mathrm{~V}$ Vydelingum, ${ }^{2} \mathrm{~S}$ Faithfull. ${ }^{1}$ Faculty of Health, Mashhad University of Medical Sciences, Mashhad, Iran; ${ }^{2}$ Division of Health \& Social Care, Faculty of Health and Medical Science, University of Surrey, Guildford, Surrey, UK

Background and Objective Despite significant progress in prostate cancer research over the last decades, screening of the disease has remained controversial. From a socio-epidemiological perspective, little is known of patients' beliefs about their illness and why they often delay in seeking diagnosis. The purpose of this qualitative study was to understand the experiences and perceptions of men about the early detection of prostate cancer.

Method This study used a grounded theory approach incorporating the theoretical perspective of social constructionism. A purposive sampling of 24 men from public and private sector hospitals who 